\title{
Influence of the factors of maternal milieu on taste preferences and metabolic parameters in mouse male and female offspring
}

\author{
Elena Denisova \\ Laboratory of Physiological Genetics \\ Institute of Cytology and Genetics SB \\ RAS Novosibirsk, Russia
}

\author{
Elena Makarova \\ Laboratory of Physiological Genetics \\ Institute of Cytology and Genetics SB \\ RAS Novosibirsk, Russia
}

\author{
Maria Savinkova \\ Department of Physiology \\ Novosibirsk State University \\ Novosibirsk, Russia
}

\begin{abstract}
Keywords - leptin, developmental programming, obesity, taste preferences
\end{abstract}

\section{Introduction}

Obesity is now a leader among noncommunicable diseases. Calorie overload induces the obesity development, and the preference and availability of sweet and fat foods contribute to obesity spread. It was shown that prenatal and early postnatal conditions affect the susceptibility to obesity and may influence on taste preferences [1], however, the mechanisms mediating maternal effects on taste preferences in offspring are unknown. The adipose tissue hormone leptin may be one of the factors mediating maternal influence on offspring phenotype. It was shown that an increase in the level of leptin in the blood of pregnant mice counteracts the development of diet-induced obesity in the offspring [2, 3], and the programming leptin effects may be different in the offspring of different sexes. It is not known whether the beneficial programming effect of maternal leptin on the susceptibility to obesity is associated with its effect on taste preferences. The aim of this study was to evaluate the effect of leptin administration to pregnant mice on metabolic phenotype, the rate of diet-induced obesity and taste preferences in offspring of different sexes.

\section{Methods}

C57BL/6J mice were housed under a 12:12-h light-dark regime and were provided ad libitum access to commercial mouse chow and water. The females were mated with the males at 12-15 weeks of age and were housed individually from the day a copulatory plug was detected (gestational day 0 , GD0). The females received subcutaneous injections of recombinant murine leptin $(2.0 \mu \mathrm{g}$ per g BW) during 3 days of pregnancy (GD11, GD12, GD13). Female body weight (BW) and food intake (FI) was measured daily during all period of pregnancy. At birth, the pups were weighed, and the litters that contained more than seven pups were reduced to seven pups. The pup BWs on postpartum days $1,7,14,21$, and 28 were measured. On postpartum day 28 , one male and one female from each litter were separated from their mothers and housed individually, and their BWs and FI were measured once a week until the age of 10 weeks. During this period, all animals were fed a standard chow diet ad libitum. From the age of 10 weeks the mice began to receive sweet biscuits and lard in addition to the standard chow. This mixture mimics the cafeteria diet and potentiates the rapid development of obesity in mice [5]. Mouse BWs and consumption of biscuits, lard and standard chow were measured over the course of 10 weeks. At the end of the experiment, the animals were sacrificed by decapitation, and samples of liver were collected to measure gene expression.

\section{Results}

Leptin administration to pregnant mice reduced their FI by $18-20 \%$, and did not affect their BWs and fetal viability, as average litter size in females treated with leptin or saline did not differ. Leptin administration did not affect the weight of pups during lactation, but had an impact on BW and FI in female offspring on a standard diet, and in male offspring on a sweet and fat diet. Beginning from the age of 8 weeks (the age of sexual maturation), the females born to leptin-treated mothers consumed less standard food and gained less weight than the female born to saline-treated mothers (repeated measures ANOVA, $\mathrm{P}<0.05$ ). However, female offspring did not differ in the rate of development of diet-induced obesity, and the body weight differences observed previously were maintained when female offspring consumed sweet and fat food. Leptin administration did not affect FI and BW in male offspring on a standard diet, and decreased the rate of weight gain on sweet and fat diet (repeated measures ANOVA, P $<0.05$ ), Fig. 1. These results are consistent with previous reports $[2,3]$ indicating the protective effect of maternal leptin on susceptibility to diet-induced obesity in mouse offspring. 
A

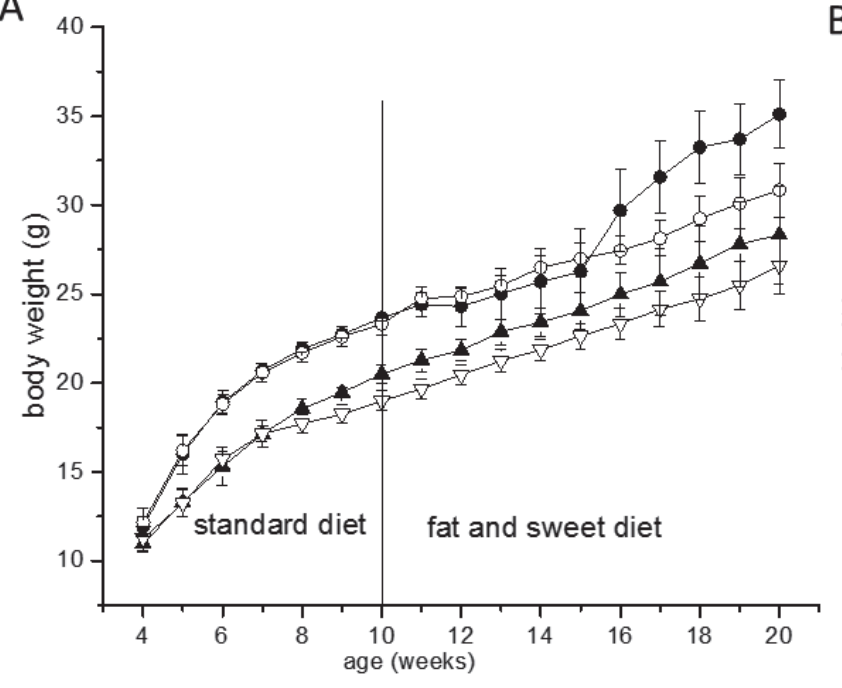

B

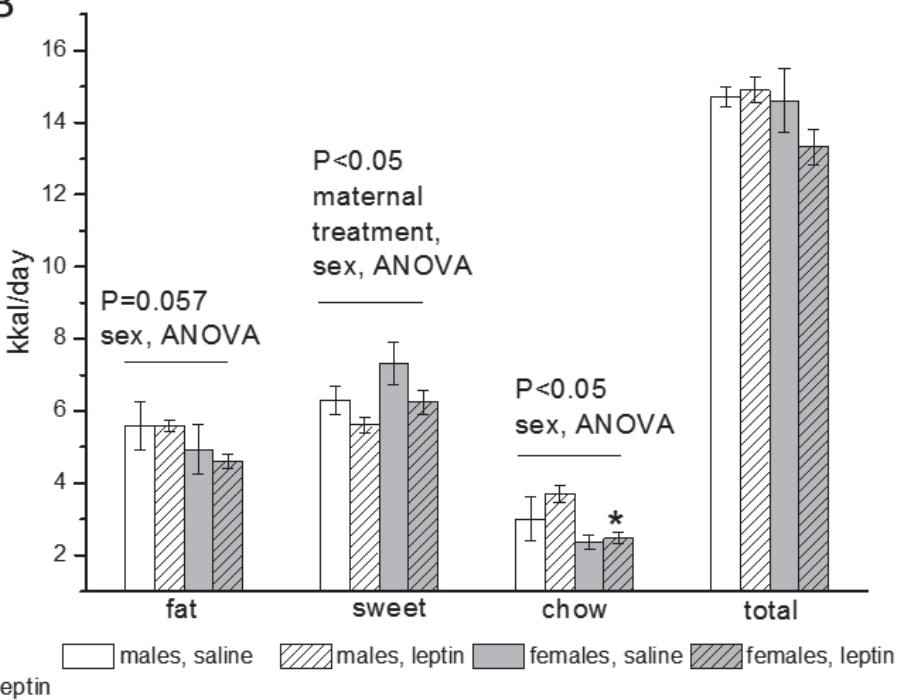

Fig. 1. Influence of leptin administration to pregnant mice on body weight changes during standard and fat and sweet diet feeding (A), and on taste preferences (B) in male and female offspring. B - Calorie intake per day from fat (lard), sweet (biscuits), and standard pelleted chow and total intake. Male and female mice born to saline- and leptin-treated mothers were fed with standard diet until the age of 10 weeks and with high-calorie fat and sweet diet after that during 10 weeks. The results are presented as an arithmetic mean $\pm \mathrm{SE}$. $* \mathrm{P}<0.05$ between males and females born to leptin-treated mothers, Post Hoc Duncan test

When fed a sweet and fat diet, males and females differed in the taste preferences: females consumed less standard pelleted chow and more sweet biscuits than males (ANOVA, $\mathrm{P}<0.05$ ). Leptin administration to pregnant mothers reduced the consumption of sweet biscuits in offspring of both sexes (ANOVA, $\mathrm{P}<0.05$ ), but did not significantly affect the calorie intake (Figure 1B). The results suggest that programming of taste preferences may be one of the mechanisms mediating the influence of maternal leptin on susceptibility to obesity in progeny, however, this issue requires further study, since no maternal effect on the energy intake has been found.

In the offspring of saline-treated mothers, sex differences in the liver expression of Fgf21 gene were observed. Fgf21 mRNA level was higher in males, which is in line with the data obtained earlier in mice with diet-induced obesity $[4,5]$. Leptin administration to pregnant mice reduced the level of FGF21 mRNA in male offspring, and sex differences in Fgf21 expression disappeared. FGF21 is one of the potent regulators of carbohydrate and lipid metabolism and insulin sensitivity. The results suggest that maternal leptin can differently effect the metabolism of male and female offspring via sex-specific programming of FGF21 function.

\section{Conclusion}

Increased level of leptin during pregnancy can be a factor that reduces the risk of diet-induced obesity in offspring of both sexes, but the mechanisms mediating programming effect of leptin can vary in offspring of different sexes. The long-lasting effect of maternal leptin on offspring taste preferences and liver FGF21 expression may be one of the reasons for its beneficial effect on the susceptibility to obesity in offspring.

\section{ACKNOWLEDGMENT}

The study was supported by Russian Foundation for Basic Research (project 17-04-01357-a and 20-015-00469-a) and Budget project 03424-2019-0041-c-01.

\section{REFERENCES}

[1] B. S. Muhlhausler and Z. Y. Ong, "The fetal origins of obesity: early origins of altered food intake", Endocr Metab Immune Disord Drug Targets, vol. 11(3), pp. 189-197, Sep 2011.

[2] E. N. Makarova, E. V. Chepeleva, P. E. Panchenko, and N. M. Bazhan, "Influence of abnormally high leptin levels during pregnancy on metabolic phenotypes in progeny mice". Am J Physiol Regul Integr Comp Physiol., vol.305, pp. R1268-R1280, 2013.

[3] K. E. Pollock, D. Stevens, K. A. Pennington, R. Thaisrivongs, J. Kaiser, M. R. Ellersieck, D. K. Miller, and L. C. Schulz, "Hyperleptinemia during pregnancy decreases adult weight of offspring and is associated with increased offspring locomotor activity in mice", Endocrinology, vol. 156, pp. 3777-3790, 2015.

[4] N. Bazhan, T. Jakovleva, N. Balyibina, A. Dubinina, E. Denisova, N. Feofanova, and E. Makarova, "Sex dimorphism in the Fgf21 gene expression in liver and adipose tissues is dependent on the metabolic condition", Online J. Biol. Sci., vol. 19(1), pp. 28-36, 2019.

[5] F. R. S. Gasparin, F. O. Carreño, J. M. Mewes, E. H. Gilglioni, C. L. S. Pagadigorria, M. R. M. Natali, K. S. Utsunomiya, et al., "Sex differences in the development of hepatic steatosis in cafeteria dietinduced obesity in young mice", Biochim. Biophys. acta. Mol. Basis Dis., vol. 1864(7), pp. 2495-2509, 2018. 